\title{
Percepción sobre el currículo de la Escuela de auxiliares de enfermería del Hospital Militar Central (Bogotá) por parte de sus estudiantes, docentes, directivos y egresados
}

\author{
Perception about the Curriculum of the School of \\ Nursing Assistants of the Central Military Hospital \\ (Bogotá) by their Students, Teachers, Directors \\ and Graduates
}

Marian Elena Ponce Sandoval* y John Jairo Briceño Martínez***

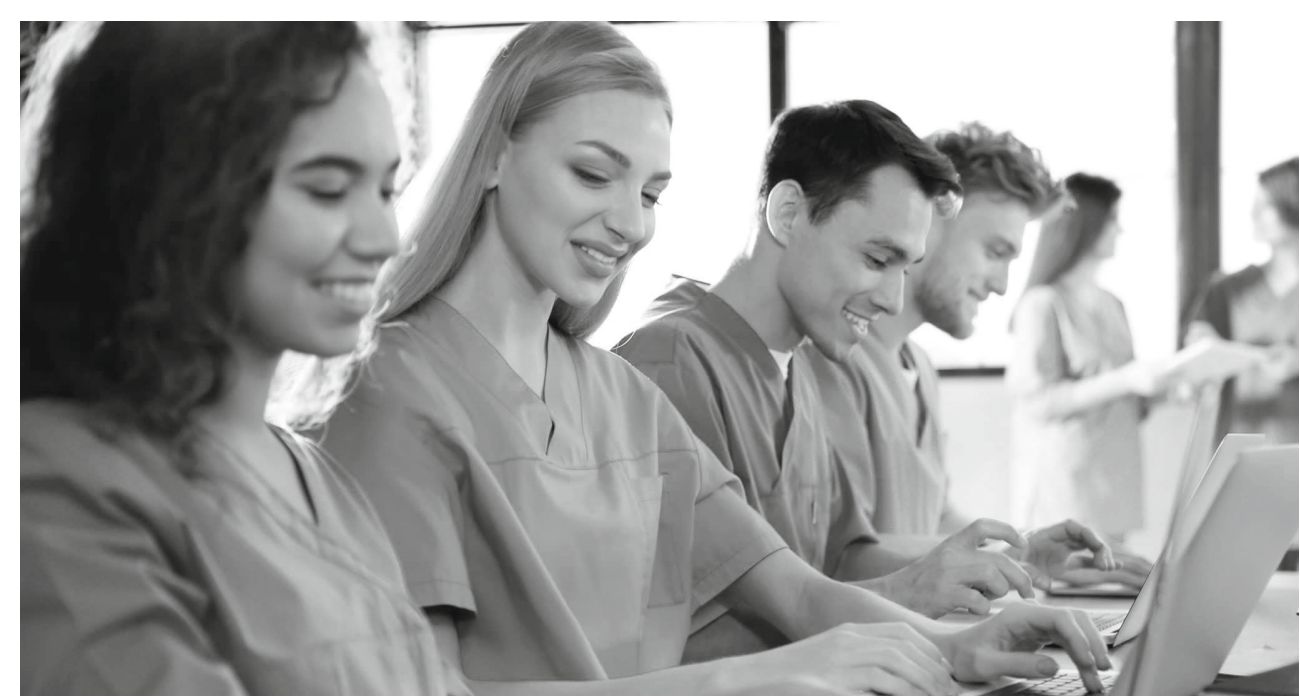

Citar este artículo como: Ponce Sandoval, M. E. y Briceño Martínez, J. J. (2020). Percepción sobre el currículo de la Escuela de auxiliares de enfermería del Hospital Militar Central (Bogotá) por parte de sus estudiantes, docentes, directivos y egresados. Revista Papeles, 12(23), 57-69.

Fecha de recibido: 10 de marzo del 2020 Fecha de aprobado: mayo 25 de 2020

* Enfermera egresada de la Universidad de Cartagena, con amplia experiencia en el área del cuidado crítico pediátrico. Con estudios de posgrado en cuidado intensivo pediátrico en la Universidad de la Sabana. Docente universitaria en la Universidad Militar Nueva Granada y magíster en Educación de la UAN. Actualmente se desempeña como enfermera líder del área de procesos y procedimientos de enfermería del Hospital Militar Central, donde labora desde hace 17 años. La autora agradece a todas las docentes, los directivos y estudiantes de la Escuela de Auxiliares de Enfermería del Hospital Militar Central por abrir las puertas de la institución y por su compromiso con la mejora de la calidad del proceso de enseñanza y aprendizaje. Correo electrónico: mponce22@uan.edu.co

*: Decano de la Facultad de Educación de la Universidad Antonio Nariño. Miembro del Grupo de Investigación Culturas Universitarias. Doctor en Tendencias y Aplicaciones de la Investigación Educativa por la Universidad de Granada (España). Correo electrónico: decano.educacion@uan.edu.co 


\title{
Resumen
}

Uno de los principales desafíos para las instituciones de formación para el trabajo es garantizar una educación pertinente y de calidad; en esa tarea, el currículo se convierte en un proceso fundamental para alcanzar esos retos. De allí que el objetivo principal del presente estudio sea conocer cuál es la percepción que tienen acerca del currículo los miembros de la comunidad académica de la Escuela de auxiliares de enfermería del Hospital Militar Central (Bogotá). La metodología tiene un enfoque cuantitativo, con un diseño no experimental y se ubica en los estudios de tipo diagnóstico. Se contó con la participación de 70 estudiantes, 21 egresados, 9 docentes y 2 directivos del centro educativo. El instrumento utilizado permite evaluar diferentes dimensiones del currículo, ya que cuenta con consistencia interna y fiabilidad de los resultados obtenidos. El análisis de las dimensiones evaluadas permite concluir que hay un consenso muy alto de percepción de satisfacción de los miembros de la comunidad educativa de la Escuela de auxiliares de enfermería del Hospital Militar Central.

Palabras clave: evaluación curricular, enfermería, calidad percibida.

\begin{abstract}
One of the main challenges for job training institutions is to guarantee a pertinent and quality education. In reaching such task, the curriculum becomes a fundamental process to achieve those challenges. Hence, the main objective of this study is to know what is the perception about the curriculum by the academic community from the School of Nursing Assistants of the Central Military Hospital (Bogotá). The methodology has a quantitative approach, with a non-experimental design and is located in diagnostic studies. It was attended by 70 students, 21 graduates, 9 teachers and 2 directors of the educational center. The instrument used allows evaluating different dimensions of the curriculum, counting on internal consistency and reliability of the results obtained. The analysis of the dimensions also evaluated allows us to conclude that there is a very high consensus of perception of satisfaction among the members of the educational community of the School of Nursing Assistants of the Central Military Hospital.
\end{abstract}

Keywords: Curricular Evaluation, Nursing, Perceived Quality.

\section{Introducción}

En Colombia, la formación en enfermería se imparte en dos niveles: profesional y técnico, de los cuales, el último se realiza en instituciones para el trabajo y desarrollo humano, debidamente reconocidas y autorizadas por la respectiva secretaría de educación territorial. Así, mediante el programa de Técnico Laboral en Auxiliar de Enfermería, cada estudiante es certificado como Técnico Laboral por Competencias en Auxiliar de Enfermería (Decreto Nacional 3616, 2005).
El fundamento de la educación para el trabajo y desarrollo humano está orientado a desarrollar y fortalecer las competencias, actitudes y valores en un individuo para desempeñarse en una actividad productiva, a fin de asegurarle la inserción exitosa al mercado laboral.

En virtud de ello, el Estado colombiano, a través del Ministerio de Educación Nacional (2008), estableció unos criterios de calidad que deben cumplir todas las instituciones

Universidad Antonio Nariño • Facultad de Ciencias de la Educación 
que oferten programas de educación para el trabajo en términos de metodología de enseñanza, evaluación, infraestructura, perfil de los docentes, recursos tecnológicos, didácticos y humanos indispensables para la relación docencia-servicio, y el nuevo componente curricular denominado competencias (Ministerio de Educación Nacional, 2008).

La formación del talento humano en salud ha sido influenciada por algunas asociaciones internacionales como la Organización Panamericana de la Salud (OPS) y la Organización Mundial de la Salud (OMS), que en múltiples oportunidades han requerido que todos los actores del sector de la salud den prioridad a la planeación de estrategias orientadas hacia la formación de su propio personal de salud, a fin de disponer de un recurso humano capacitado para conseguir, mantener y asegurar la cobertura sanitaria de calidad para todos los habitantes (OPS, 2005).

El talento humano es, entonces, un elemento indispensable para asegurar el éxito de todo sistema de salud, y, por lo tanto, cualquier situación que afecte su formación impactará necesariamente en la calidad del sistema (OMS, 2016).

Esta preocupación obedece no solo a la importancia que tiene este grupo dentro del sistema de salud, por ser el principal proveedor del servicio, sino también porque representa el mayor gasto en el mismo sistema, el cual es un componente básico en el proceso de formulación, aprobación, ejecución y seguimiento de políticas públicas en el marco de los sistemas de salud (Cañizares y García, 2015).

En Colombia, a partir de la puesta en marcha de la reforma del sector de la salud en 1993, se implementó una serie de políticas encaminadas a garantizar una formación integral y de calidad para los trabajadores de la salud, entre las que se destaca el establecimiento de unos perfiles ocupacionales para los auxiliares de enfermería basados en competencias laborales, donde se enuncian explícitamente los conocimientos, habilidades y destrezas que debe adquirir un auxiliar de enfermería para brindar un cuidado con calidad científica y humana.

Adicionalmente, se cuenta con la implementación de un sistema obligatorio de garantía de calidad, no solo para las instituciones educativas sino también para las instituciones de salud (Ministerio de Educación Nacional, 2008).

Ahora bien, según la Organización de las Naciones Unidas para la Educación, la Ciencia y la Cultura (UNESCO), el currículo juega un papel fundamental en la calidad educativa y los criterios para valorar su calidad son la planificación de los contenidos, el desarrollo de la enseñanza, la metodología y la evaluación, que es entendida no solamente como seguimiento del aprendizaje del estudiante, sino también como medio para determinar si ese currículo realmente responde a las necesidades de la sociedad (Stabback, 2016).

De esta manera, la evaluación curricular resulta una condición necesaria para examinar la calidad de las instituciones educativas, la cual puede realizarse desde la perspectiva tradicional, es decir, en función del aprendizaje del estudiante o, bien, desde el criterio de los participantes del servicio educativo. Así se abordan aspectos como la satisfacción o la expectativa, que se entienden como el estado emocional y mental en el que se concretiza la percepción de la persona frente al cumplimiento de sus expectativas (Sánchez, Carvajal y Pérez, 2015).

El talento humano es, entonces, un elemento indispensable para asegurar el éxito de todo sistema de salud, $y$, por lo tanto, cualquier situación que afecte su formación impactará necesariamente en la calidad del sistema. 
A partir de lo anterior, en este estudio se analiza la percepción que tienen los estudiantes, profesores, docentes y directivos sobre diferentes aspectos del currículo del programa Técnico laboral por competencias en auxiliar de enfermería de la Escuela de enfermería del Hospital Militar Central, lo cual resulta pertinente no solo porque proporciona información de primera mano sobre la marcha del proceso educativo, sino también porque sirve de retroalimentación a docentes y directivos para la toma de decisiones que les permitan mejorar el proceso enseñanza-aprendizaje.
Por otra parte, si bien existe una reglamentación que incluye criterios de calidad en esta oferta educativa, resulta oportuno examinar si las características del currículo de este programa se encuentran, o no, alineadas con las demandas del sector productivo, teniendo en cuenta que ninguna instancia del sistema de garantía de calidad para las instituciones de educación para el trabajo incluye evaluaciones sobre la calidad de los aprendizajes o pertinencia curricular (Gonzáles y Rosas, 2016).

\section{Aproximación histórica a la formación de auxiliares de enfermería}

La formación en enfermería ha estado marcada por el rol protagónico de las mujeres que ocurría dentro de los hospitales y que estaba a cargo de médicos o religiosos; a ello se suma que la instrucción que se daba a las aprendices no contaba con un currículo establecido, y era el profesional en medicina quien decidía el contenido teórico que debían aprender las estudiantes para que ejercieran el papel de asistentes o ayudantes (Álvarez, 2015).

Otro aspecto importante que marcó los inicios de la enfermería fue la participación de las mujeres en los conflictos bélicos a principios del siglo XX en Europa, tanto en labores asistenciales con los heridos como en el mantenimiento de los hospitales militares y de la Cruz Roja.

Se reconoce claramente la Escuela para Enfermeras Nightingale como la primera escuela de formación en enfermería, cuyo modelo pedagógico estaba fundamentado en un currículo tradicional en el que las enfermeras aprendían haciendo cada uno de los procedimientos correspondientes para el cuidado de los pacientes.

El aporte fundamental de Florence Nightingale (1991) a la enfermería fue la introducción de los conceptos de normas para el control de infecciones, administración hospitalaria, el cuidado como eje fundamental y la importancia del ambiente en el bienestar físico y mental de los individuos.

En toda Europa, las escuelas de enfermería eran, en sus inicios, dependientes de los hospitales donde se hacía docencia y se prestaba el servicio de salud; los cursos se realizaban por iniciativa de médicos o religiosas, y bajo este mismo modelo educativo se establecieron también las escuelas de enfermería en Estados Unidos (López Vallecillo, 2016).

De acuerdo con Ponti (2016), dos elementos caracterizaron la formación en enfermería a nivel de Latinoamérica: por una parte, se dio bajo la influencia de las congregaciones religiosas dedicadas a la enseñanza de actividades relacionadas con el cuidado de pacientes, orientada a mujeres de buena voluntad y con vocación de servicio, mediante un modelo conductista en el que la estudiante aprendía los procedimientos básicos de enfermería por imitación.

Por otra parte, el director de la escuela era un profesional médico, quien se encargaba de seleccionar el contenido teórico de la 
enseñanza y de impartirlo a través de clases teóricas magistrales, en las que la estudiante, de manera pasiva, estaba atenta a todas las explicaciones.

En el caso colombiano, el ejercicio de la enfermería coincidió con la llegada de la congregación religiosa de los Hermanos de San Juan de Dios, en 1564, quienes estaban a cargo del Hospital San Pedro, y quienes, aunque no eran enfermeros, sí conocían el oficio porque durante los conflictos armados en Europa los monasterios eran usados para la asistencia de los heridos.

Durante la época colonial, se resalta la influencia del sacerdote Pedro Claver, quien se dedicó al cuidado de enfermos de lepra y para muchos es reconocido como el padre de la enfermería en Colombia (Gómez Serrano, 2011).

Entre 1935 y 1954, ante la necesidad de dar cobertura a los servicios hospitalarios, debido a la escasez de enfermeras profesionales, que para ese momento centraban sus actividades en labores administrativas, se reconocieron las auxiliares de enfermería como miembros del equipo de salud y era el Ministerio de Educación el responsable de establecer los requisitos para el funcionamiento de las escuelas para auxiliares de enfermería.

Se definió el auxiliar de enfermería como la persona capacitada por medio de un programa educativo oficial para proporcionar actividades de cuidado básico a los sujetos y comunidades, siempre bajo la orientación y supervisión de profesionales del área de la salud, médicos o enfermeros.

La duración del curso era mínimamente de 9 meses, 1440 horas aproximadamente, de las cuales una tercera parte se utilizaba para la enseñanza de los aspectos teóricos del programa y el resto a la práctica formativa en los hospitales en los diferentes turnos, para que los auxiliares adquirieran la experiencia de las diferentes rutinas.

El programa educativo incluía dos áreas que eran educación general, cuyo contenido académico incluía ética, comunicación, motivación, trabajo en equipo y relaciones interpersonales; y otra área de educación de la enfermería, con asignaturas como anatomía, higiene, cuidado de los enfermos, nociones de farmacia, nutrición y masajes (Nájera y Castrillón, 2011).

Hoy la enseñanza de la enfermería a nivel técnico hace parte de la oferta educativa de las instituciones para el trabajo y el desarrollo humano, antes educación no formal, y de acuerdo con el Ministerio de Educación nacional (2017), está reglamentada por la Ley 1064 de 2006. El objetivo de esta modalidad es capacitar a las personas para ingresar al mercado laboral desarrollando competencias laborales específicas.

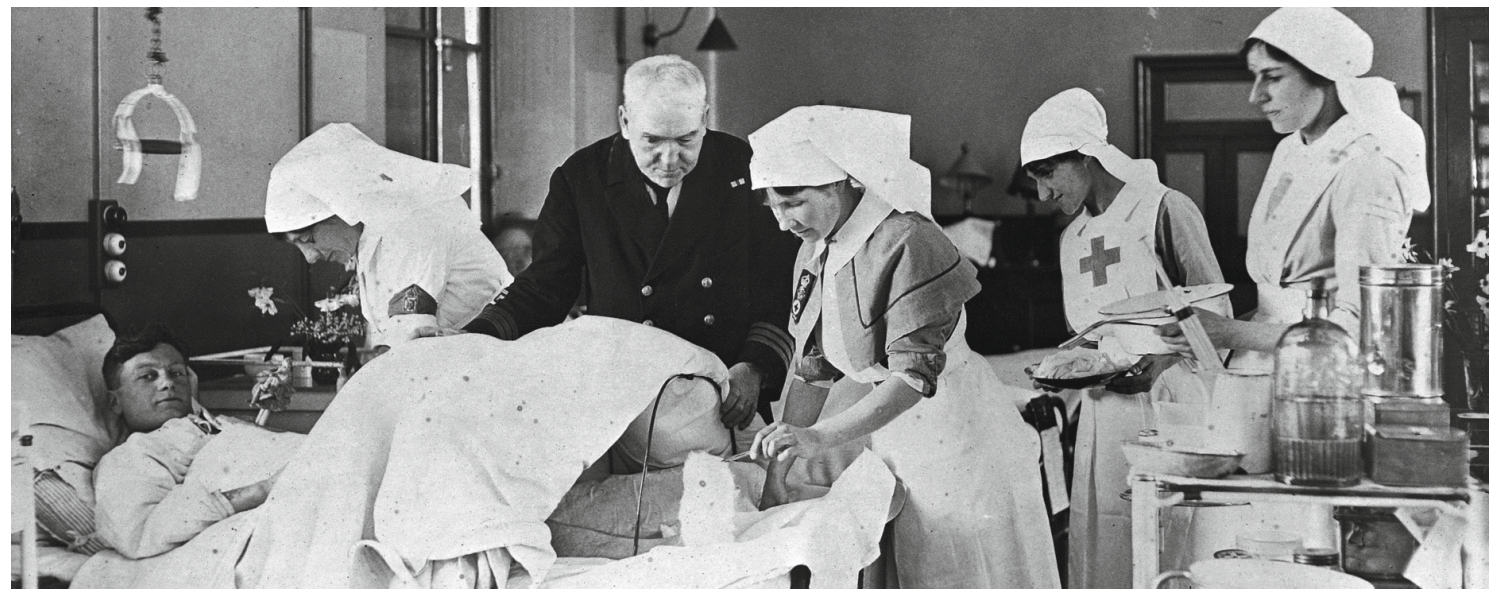

Revista PAPELES • ISSN 0123-0670 • Vol. 12(23) • pp. 57-69 • Enero-junio de 2020 


\section{Escuela de auxiliares de enfermería del Hospital Militar Central}

El Hospital Militar Central es una institución prestadora de salud que brinda servicios de alta y mediana complejidad a los usuarios del Subsistema de Salud de las Fuerzas Militares (ejército, armada y fuerza aérea).

En el ámbito académico, cuenta con convenios de docencia y servicio con distintas universidades nacionales e internacionales, que es el centro de prácticas para estudiantes pertenecientes a diferentes programas académicos del área de la medicina a nivel de pregrado y postgrado, avalados por la Universidad Militar Nueva Granada. Tiene, además, su propia escuela de auxiliares de enfermería, la cual inició labores en el año 1958, con el propósito de preparar auxiliares de enfermería para las fuerzas armadas, quienes estuvieran especializados en atender a heridos en zona de combate y en evacuación de víctimas (Gómez Serrano, 2011).

De conformidad con lo establecido en el Decreto 1011 del 2006, el hospital se acogió de forma voluntaria al Sistema Obligatorio de Gestión de Calidad en Salud (SOGC) y desarrolló un sistema de gestión de calidad como una herramienta estratégica para el mejoramiento continuo de sus procesos.

La Escuela de auxiliares de enfermería del Hospital Militar Central es una institución de formación para el trabajo y el desarrollo humano, que, como establecimiento educativo, ofrece su servicio no solo al gremio militar sino también a la comunidad civil por medio del programa académico de Técnico laboral por competencias en Auxiliar de enfermería, y su funcionamiento se da en conformidad con la normatividad vigente para este tipo de instituciones.

Su currículo es de carácter modular y está estructurado con base en las normas de competencias laborales específicas definidas por las mesas sectoriales que lidera el Servicio Nacional de Aprendizaje (SENA), luego de su entrada en vigor con el Decreto 3616 de 2005.

El programa es de modalidad presencial, se desarrolla en 1800 horas, de las cuales el 40\% corresponden a la teoría y el $60 \%$ a la práctica, y que se llevan a cabo en escenarios reales del entorno productivo a través de convenios de tipo educativo y de servicios.

Se plantea una educación basada en competencias, apoyada en el modelo pedagógico constructivista. Por la naturaleza propia del quehacer del auxiliar de enfermería, hay un énfasis en el saber hacer, dado que lo prioritario para su perfil ocupacional es el desarrollo de habilidades y destrezas para brindar una atención segura a todos los pacientes. Esta característica conlleva a la adopción de metodologías activas para el aprendizaje y al reconocimiento del estudiante como el actor principal de su proceso educativo.

Los componentes básicos del currículo del programa están organizados en tres niveles formativos, con sus respectivas asignaturas, según las competencias que se desarrollan de manera secuencial en cada etapa del proceso educativo.

Todas las asignaturas de contenido teóricopráctico incluyen trabajos en el laboratorio, donde se utilizan metodologías basadas en aprendizajes activos e independientes, tales como el aprendizaje basado en problemas (ABP), en el que, a partir de situaciones problémicas, el estudiante no solo aplica los protocolos o manejos aprendidos en el aula, sino que también aprende a buscar información en las distintas fuentes, a aplicarla para solucionar la situación propuesta y facilitar, finalmente, la toma de decisiones en un escenario real (Roca, Reguant y Canet, 2015). 


\section{Generalidades del currículo y de la evaluación curricular}

Se dispone de una gran variedad de definiciones sobre el currículo. De acuerdo con Luna y López (2012), la palabra currículo proviene del latín currere, que en español significa "recorrer un camino", y representa, por un lado, lo que una persona ha estudiado a lo largo de su vida y se conoce como currículum vitae, y, por otro lado, es el recorrido que deben realizar los estudiantes durante la construcción de una carrera. Así, tiene que ver con los contenidos que el profesor debe enseñar y que los estudiantes deben aprender y marca el camino de la labor del profesor y del estudiante en función de alcanzar los objetivos planteados.

El currículo tiene las funciones de manifestar las metas del sistema educativo, de guiar a los docentes para conseguir esas metas y de actuar como referente para la evaluación de calidad del sistema educativo. Esta noción da cuenta de la capacidad reguladora que desde el principio ha tenido el currículo en todas las actividades involucradas en el proceso de enseñanza-aprendizaje (Luna y López, 2012).

Por su parte, en la Ley 115 de 1994 (Artículo 76) se define el currículo como:

el conjunto de criterios, planes de estudio, metodologías y procesos que contribuyen a la formación integral y a la construcción de la identidad cultural nacional, regional y local, incluyendo también los recursos humanos, académicos y físicos para poner en práctica las políticas y llevar a cabo el proyecto educativo institucional.

Más allá de la definición, el currículo es el punto de unión de la escuela con la sociedad y sus demandas e intereses, y refleja los propósitos educativos de esta sociedad (Malagón-Plata, 2008). De esta manera, un currículo de calidad es aquel que se evalúa de manera sistemática y planificada, es decir: basado en una finalidad y un alcance claramente definido y a distintos niveles del sistema educativo, utilizando datos válidos y fidedignos, en un claro marco de calidad, de manera periódica y por personas debidamente calificadas y experimentadas (Stabback, 2016, p. 38).

En este contexto, la evaluación curricular y la evaluación de las instituciones educativas se erigen en estrategias para mejorar la calidad de la educación (Brovelli, como se cita en Salas, 2016).

La evaluación curricular es, entonces, el proceso que permite corroborar en qué medida el currículo satisface las demandas de la sociedad; lo que implica el análisis de todas las actividades y procesos diseñados por la institución para el aprendizaje de los estudiantes. Se trata de una actividad indispensable para favorecer el nivel de calidad de la educación, la cual puede ser observada desde los distintos puntos de vista de la comunidad educativa (Brovelli, como se cita en Salas, 2016).

Leipiani (2013) expresa que una manera de evaluar la calidad educativa es por medio de la satisfacción del estudiante, lo cual está

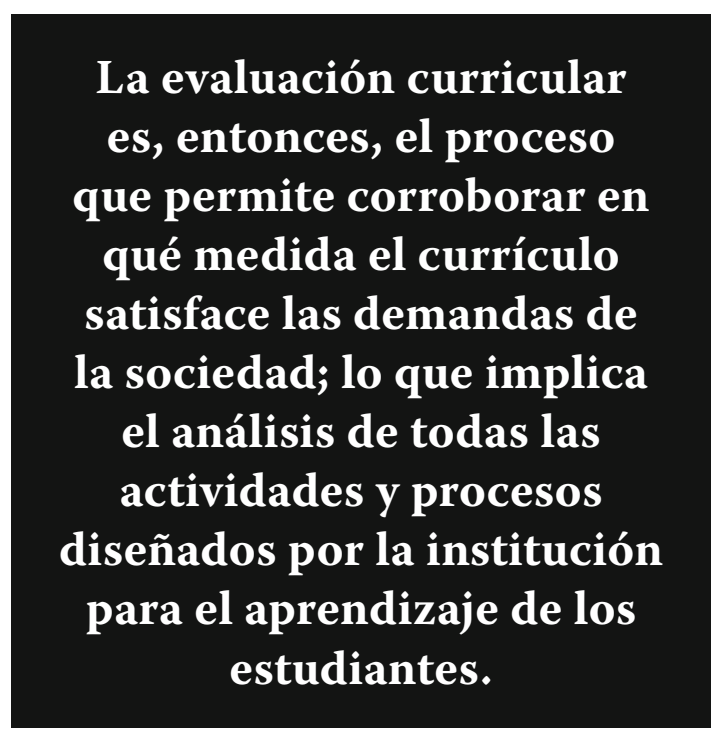


altamente relacionada con conceptos como la calidad de un servicio y la satisfacción del cliente. De esta forma, propone que la percepción de la calidad repercute en la satisfacción del cliente, lo que también se aplica a la satisfacción académica, cuyo aporte es identificar oportunidades de mejoramiento para aumentar la satisfacción del estudiante respecto a la acción educativa del centro.
Basado en lo anterior, en este trabajo, que un miembro de la comunidad educativa de la Escuela de auxiliares de enfermería del Hospital Militar Central tenga percepción favorable respecto de cualquier aspecto indagado se considera un indicador eficaz de la calidad de esa dimensión curricular y, por ende, de la institución de enseñanza (Jiménez, Terriquez, Carrillo y Robles, 2011).

\section{Materiales y método}

La metodología de este estudio es de tipo cuantitativo-descriptivo y se ubica en los estudios de tipo diagnóstico. Se realiza con 70 estudiantes que cursan el I y II módulo del programa académico Técnico laboral por competencias en Auxiliar de enfermería, 21 egresados que laboran actualmente en el servicio de pediatría del Hospital Militar Central, 9 docentes de la Escuela de auxiliares de enfermería, y 2 directivos, que son la directora y la coordinadora académica del mismo programa.

Para lograr los objetivos planteados se aplican a los estudiantes y egresados cuatro cuestionarios de autocumplimentación, adaptados de la Vicerrectoría de Investigación de la Universidad de Jaén (Universidad de Jaén, 2009). Además, esta herramienta fue adaptada y utilizada para evaluar la percepción del currículo de medicina de una universidad de Colombia (Orozco, 2017).

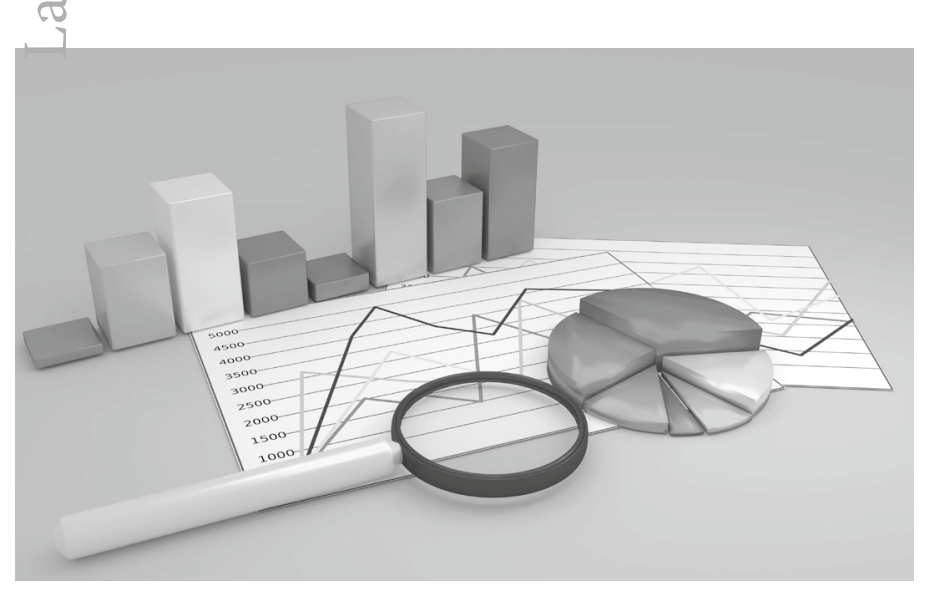

El cuestionario comprende 73 ítems que indagan sobre 6 aspectos del currículo: objetivos del plan de estudios, planificación de la enseñanza, admisión del estudiante, orientación al estudiante, recursos y servicios y personal académico.

El cuestionario para docentes y para directivos contiene 68 ítems que indagan sobre 7 aspectos curriculares: plan de estudios y contenidos programáticos, planificación de la enseñanza, desarrollo de la enseñanza y evaluación de aprendizajes, orientación al estudiante, personal académico, recursos y servicios y estudiantes.

Cada ítem corresponde a una escala de Likert de 5 opciones, en las que $4=$ Muy de acuerdo, 3 = De acuerdo, 2 = En desacuerdo, 1 = Totalmente en desacuerdo y $0=$ No sabe $/$ No contesta.

El instrumento dispone, además, de un apartado de "observaciones" en el que el encuestado puede expresar sus opiniones respecto de la pregunta. La toma de la información se realizó en las instalaciones de la Escuela de Auxiliares de Enfermería, en dos sesiones, cada una de 40 minutos.

Los datos se procesaron con el programa estadístico STATA, y se usó la estadística descriptiva para realizar análisis de frecuencias acumuladas y porcentajes; la fiabilidad de los instrumentos es obtenida con el alfa de Cronbach. 


\section{Resultados}

\section{Análisis descriptivo de los estudiantes}

\section{Características sociodemográficas}

El 53\% de los estudiantes cursa el módulo II y el $47 \%$ el módulo I; el $60 \%$ es de sexo masculino y el $40 \%$ femenino; la edad promedio de los encuestados es de 26.9 años ( $\mathrm{DE}=10.4$ años): la edad mínima es de 17 años y la máxima de 40; el 57\% de la muestra de estudiantes es soltero, $32 \%$ casado, el $7 \%$ mantiene una unión libre y el 3\% está divorciado.

Respecto a la variable de vinculación a las fuerzas militares, el $46 \%$ de los estudiantes pertenecía a las fuerzas militares y el $54 \%$ restante era personal civil. La fuerza con mayor representación es el ejército con 26 estudiantes, que corresponden al 37\%; sigue la armada nacional con 4 estudiantes (6\%), y la fuerza aérea con 2 estudiantes (3\%).

\section{Percepción general de los estudiantes respecto al currículo}

El porcentaje de satisfacción obtenido en cada una de las dimensiones indagadas es: objetivos del plan de estudio (91.21\%), planificación de la enseñanza (89.01\%), admisión del estudiante $(90.11 \%)$, orientación al estudiante (83.52\%), personal académico (93.41\%) y recursos y servicios (81.32\%).

La dimensión personal-académica resultó con una satisfacción baja $(79,12 \%)$ en comparación con las otras, aunque, en general, se evidencia un consenso muy alto de satisfacción de los estudiantes con el programa.

\section{Análisis descriptivo de los egresados}

\section{Características sociodemográficas}

Las principales características sociodemográficas de la población de 21 egresados son
Tabla 1. Percepción de los estudiantes para cada dimensión curricular

\begin{tabular}{|l|c|c|c|}
\hline \multicolumn{1}{|c|}{ Dimensión } & N & Frecuencia & $\begin{array}{c}\text { \% de } \\
\text { satisfacción }\end{array}$ \\
\hline $\begin{array}{l}\text { 1. Objetivos del } \\
\text { plan de estudios }\end{array}$ & 70 & 67 & $95,71 \%$ \\
\hline $\begin{array}{l}\text { 2. Planificación } \\
\text { de la enseñanza }\end{array}$ & 70 & 67 & $95,71 \%$ \\
\hline $\begin{array}{l}\text { 3. Admisiones de } \\
\text { los estudiantes }\end{array}$ & 70 & 68 & $97,14 \%$ \\
\hline $\begin{array}{l}\text { 4. Orientación de } \\
\text { los estudiantes }\end{array}$ & 70 & 65 & $92,86 \%$ \\
\hline $\begin{array}{l}\text { 5. Personal } \\
\text { académico }\end{array}$ & 70 & 66 & $94,29 \%$ \\
\hline $\begin{array}{l}\text { 6. Recursos y } \\
\text { servicios }\end{array}$ & 70 & 66 & $94,29 \%$ \\
\hline
\end{tabular}

Fuente: elaboración propia

las siguientes: el $100 \%$ es de sexo femenino, el tiempo de egreso promedio es de 15.8 años ( $\mathrm{DE}=11.25$ años). El egresado más reciente se graduó hace 6 meses y el más antiguo hace 32 años. El $23 \%$ es de estado civil soltero (5 egresados) y el $77 \%$ casado.

\section{Percepción general de los egresados res-} pecto al currículo

El porcentaje de satisfacción obtenido en cada una de las dimensiones examinadas es: objetivos del plan de estudio (85.71\%), planificación de la enseñanza (76.19\%), admisión del estudiante (85.71\%), orientación al estudiante (95.24\%), personal académico $(90.48 \%)$ y recursos y servicios $(66.67 \%)$.

La dimensión de recursos y servicios resultó con una satisfacción baja $(66,67 \%)$ con relación a las otras, aunque, en general, se evidencia un consenso muy alto de satisfacción de los egresados con el programa. 
Tabla 2. Percepción de egresados para cada dimensión curricular

\begin{tabular}{|l|c|c|}
\hline \multicolumn{1}{|c|}{ Dimensión } & N & \% de satisfacción \\
\hline $\begin{array}{l}\text { 1. Objetivos del plan } \\
\text { de estudios }\end{array}$ & 21 & $85,71 \%$ \\
\hline $\begin{array}{l}\text { 2. Planificación de la } \\
\text { enseñanza }\end{array}$ & 21 & $76,19 \%$ \\
\hline $\begin{array}{l}\text { 3. Admisiones de los } \\
\text { estudiantes }\end{array}$ & 21 & $85,71 \%$ \\
\hline $\begin{array}{l}\text { 4. Orientación de los } \\
\text { estudiantes }\end{array}$ & 21 & $95,24 \%$ \\
\hline 5. Personal académico & 21 & $90,48 \%$ \\
\hline 6. Recursos y servicios & 21 & $66,67 \%$ \\
\hline
\end{tabular}

Fuente: elaboración propia

\section{Análisis descriptivo de los docentes y directivos}

\section{Características sociodemográficas}

El estudio se realizó con 9 docentes y 2 directivos, el $100 \%$ de sexo femenino. El cuerpo docente es joven y la media de edad es de 40.1 años; son profesionales experimentadas, con un tiempo laboral promedio de 13.3 años.

\section{Percepción general de los docentes y directivos respecto al currículo}

Los docentes y directivos evalúan todas las dimensiones curriculares por encima del punto medio de satisfacción, lo que conlleva a unos valores altos de satisfacción y un consenso muy alto de esta población.

De esta manera, se puede ver que en su totalidad esta población se encuentra de acuerdo o muy de acuerdo con los objetivos del plan de estudios, la planificación y el desarrollo de la enseñanza, así como con la orientación al estudiante, personal académico, los recursos y servicios y con sus estudiantes.

\section{Prueba de confiabilidad para el cuestionario}

La confiabilidad del instrumento fue calculada por medio del coeficiente alfa de Cronbach, el cual especifica que si el cálculo está por debajo de 0.8 llevará a conclusiones equivocadas, mientras que si está por encima de ese valor y muy próximo a la unidad, se considera un instrumento fiable, cuyos ítems están altamente correlacionados y que mide (por medio de la escala Likert) la variable que pretende cuantificar (Hernández Sampieri, Fernández Collado y Baptista, 2014).

Los resultados para el instrumento usado en esta investigación informan un valor promedio de correlación de 0.54 , que ilustra asociación entre los ítems, mientras que el coeficiente es de 0.87 , por lo cual se considera que los hallazgos son confiables.

Tabla 3. Percepción de docentes y directivos para cada dimensión curricular

\begin{tabular}{|l|c|c|c|c|}
\multicolumn{1}{|c|}{ Dominio } & N & $\begin{array}{c}\text { Punto medio } \\
\text { de satisfacción }\end{array}$ & $\begin{array}{c}\text { \# personas } \\
\text { "satisfechas" }\end{array}$ & $\begin{array}{c}\text { \% } \\
\text { Satisfacción }\end{array}$ \\
\hline 1. Plan de estudios y sus contenidos programáticos & 11 & 15 & 11 & 100 \\
\hline $\begin{array}{l}\text { 2. Planificación de la enseñanza } \\
\text { 3. Desarrollo de la enseñanaza y evaluación de }\end{array}$ & 11 & 33 & 11 & 100 \\
\hline aprendizajes & 11 & 15 & 11 & 100 \\
\hline 4. Orientación al estudiante & 11 & 20 & 11 & 100 \\
\hline 5. Personal académico & 11 & 15 & 11 & 100 \\
\hline 6. Recursos y servicios & 11 & 30 & 11 & 100 \\
\hline 7. Los estudiantes & 11 & 43 & 11 & 100
\end{tabular}

Fuente: elaboración propia 


\section{Conclusiones}

Las dimensiones mejor calificadas en el grupo de estudiantes son objetivos del plan de estudios y personal académico; este último abarca a los profesores de clases teóricas, prácticas, prácticas clínicas y modo en que se evaluaba.

Al ser la dimensión de recursos y servicios el ítem con la percepción de satisfacción más baja, resulta indispensable mejorar los servicios administrativos, $y$, en especial, el desempeño del personal a cargo del servicio de los estudiantes y las condiciones de infraestructura, soporte tecnológico, equipamiento de laboratorios y todos los insumos con que se cuentan para el desarrollo de la enseñanza. Se trata, entonces, de oportunidades de mejora para incrementar la calidad de los procesos del centro educativo, y, por ende, elevar la percepción de satisfacción de los estudiantes.

Los estudiantes y egresados destacan positivamente el desarrollo de la docencia; esto es, los conocimientos y las orientaciones del profesor en el aula y fuera de ella, lo cual es un indicador de la calidad de la institución.

La percepción de los profesores sobre los estudiantes es buena, por lo que el 100\% manifiesta que está satisfecho con el grupo de estudiantes. De igual modo, consideran que el contenido del programa, la metodología y la evaluación están acordes con la naturaleza de los saberes y con el contexto asistencial del ejercicio de la enfermería.

En general, los miembros de la comunidad educativa se encuentran satisfechos con el servicio educativo de la escuela. Los resultados demuestran que los estudiantes, egresados, docentes y directivos expresan una percepción satisfactoria del currículo del programa Técnico laboral por competencias en Auxiliar de enfermería de la Escuela de auxiliares del Hospital Militar Central.

Pese a que ningún objetivo planteado hace referencia a la valoración de la pertinencia del programa, el estudio evidenció que el proceso educativo de la Escuela de auxiliares se encuentra alineado con la misión y la visión de la institución, lo cual concuerda con la percepción que tienen los egresados acerca de que el currículo de la escuela les brinda las herramientas para desempeñarse de manera correcta en el hospital.

De esta manera, se puede decir que hay correspondencia entre el currículo del programa y las exigencias del Hospital Militar, teniendo en cuenta que se señala la pertinencia como un aspecto muy importante en la modalidad educativa de formación para el trabajo; esto es, desarrollar las competencias laborales específicas correspondientes a las demandas del sector laboral.

Este es un hallazgo muy interesante en vista de que existe poca evidencia de que las instituciones educativas de formación para el trabajo y sus programas sean evaluadas en términos de calidad y de pertinencia (Saavedra y Medina, 2012).

Sin embargo, es importante que se incluyan actividades culturales y de atención a la diversidad en las diferentes dimensiones en que esta se expresa, por ejemplo, religiosa, sexual o cultural, con el fin de enriquecer el proceso de enseñanza de la enfermería y el horizonte cultural del estudiantado.

Estos dos aspectos deben ser revisados por las directivas de la escuela, toda vez que los estudiantes les den una valoración alta a la necesidad de la recreación; por otro lado, el tema del abordaje a la diversidad era desconocido para ellos y no disponían de la información necesaria para evaluarlo.

Los resultados obtenidos permiten a los docentes y directivos tomar decisiones para que, en determinado caso, se reestructure el trabajo académico para incrementar la competitividad de los futuros auxiliares de enfermería y asegurar el éxito en su inserción a la vida productiva. 
Dar continuidad a este tipo de estudios le dará herramientas a la escuela de enfermería para fortalecer su servicio y, por lo tanto, mejorar su calidad educativa.

En este caso, se podría optar por una metodología mixta (cuantitativa y cualitativa) para poder complementar las respuestas y profundizar en las razones que sustentan la percepción de los múltiples aspectos del currículo en los diferentes participantes, lo que permitiría obtener conclusiones más consistentes (Aguirre, 2007).

\section{Referencias}

Aguirre, E. (2007). La evaluación de la actividad docente de la Escuela de Enfermería de la Universidad de Costa Rica. Revista Educación, 31(1), 65-76.

Álvarez, D. (2015). Enfermería en América Latina: una mirada al horizonte. Avances en Enfermería, 33(2), 295-305.

Cañizares, R. y García, J. F. (2015). Planificación y gestión de recursos humanos en salud en los países andinos. Evidencias para la toma de decisiones.

Congreso de Colombia. (8 de febrero de 1994) Artículo 76 [Título IV]. Ley General de Educación. [Ley 115 de 1194]. DO: 41.214.

Gómez Serrano, C. (2011). Tres escuelas una historia La formación de enfermeras en la Universidad Nacional de Colombia 1920-1957. Avances en Enfermería, 29(1), $169-171$.

González, C. y Rosas, D. (2016). Avances y retos en la formación para el trabajo en Colombia. Bogotá: BID.

Hernández Sampieri, R., Fernández Collado, C. y Baptista Lucio, P. (2014). Metodología de la investigación. México: McGraw Hill.

Jiménez, G. A., Terriquez, B. y Robles, F. (2011). Evaluación de la satisfacción académica de los estudiantes de la Universidad Autónoma de Nayarit. Revista Fuente, 2(6), 46-56.

Leipiani, I. (2013). Satisfacción del Estudiante de Enfermería con el Proceso Formativo Adaptado al Espacio Europeo de Educación
Superior. Revista Enfermería Docente, 101, 22-28. Recuperado de https://www.huvv. es/sites/default/files/revistas/ED-101-06. pdf

López Vallecillo, M. (2016). Presencia social e imagen pública de las enfermeras en el siglo XX (1915-1940) [Universidad de Valladolid]. https://doi.org/10.35376/10324/16513

Luna, E. y López, G. (2011). El currículo: concepciones, enfoques y diseño. Revista Unimar, 58, 65-76.

Malagón Plata, L. A. (2008). El currículo: perspectivas para su interpretación. Investigación y Educación en Enfermería, 26(2).

Ministerio de la Protección Social. (10 de octubre de 2005). Establece el Sistema Obligatorio de Garantía de Calidad de la Atención de Salud del Sistema General de Seguridad Social en Salud. [Decreto 3616]. DO: 46.060 .

Ministerio de la Protección Social. (3 de abril de 2006). Perfiles ocupacionales y normas de competencia laboral para auxiliares en las áreas de salud. [Decreto 1011]. DO: 46.230 .

Ministerio de Educación Nacional. (2008). Verificación de los requisitos básicos para el funcionamiento de los programas de educación para el trabajo y el desarrollo humano. Colombia. Guía núm. 29. Recuperado de https://www.mineducacion.gov. co/1759/articles-237704_Guia29.pdf

Ministerio de Educación Nacional. (2017). Plan Nacional Decenal de Educación 
2016-2026. El camino hacia la calidad y la equidad. Colombia.

Nájera, R. M., y Castrillón, M. (2011). La Enfermería en América Latina Situación actual, áreas críticas y lineamientos para un Plan de Desarrollo. Facultad de Enfermería, Universidad de Antioquia, Medellín, Colombia.

Nightingale, F. (1991). Notes on Nursing: What it is and what is not. En J. Castro. Notas sobre enfermería qué es y qué no es. México: Salvat Editores.

Organización Mundial de la Salud. (2016). Estrategia mundial de recursos humanos para la salud: personal sanitario 2030: Proyecto de resolución presentado por las delegaciones de la Argentina, los Estados Miembros de la Unión Europea, los Estados Unidos de América, el Japón, Mozambique, Noruega, Sudáfrica, Suiza, Tailandia, Zambia y Zimbabwe (No. A69/B/CONF./4). Recuperado de http://apps.who.int/gb/ebwha/pdf_files/ WHA69/A69_38-sp.pdf.

Organización Panamericana de la Salud. (2005). A la acción Toronto. Hacia una década de recursos humanos para la salud en las Américas, OPS, Salud Canadá y el Ministerio de Salud de Ontario y Long Term Care. Toronto, Canadá.
Orozco, E. (2017). Percepciones de la comunidad académica de la Facultad de Medicina de la Universidad Antonio Nariño con respecto al currículo de la misma facultad. (Tesis de maestría). Universidad Antonio Nariño, Bogotá.

Ponti, L. (2016). La enfermería y su rol en la educación para la salud. Buenos Aires, Argentina: UAI Editorial.

Roca, J., Reguant, M. y Canet, O. (2015). Aprendizaje basado en problemas, estudio de casos y metodología tradicional: una experiencia concreta en el grado en enfermería. Procedia Social and Behavioral Sciences, 196, 163-170.

Sánchez, J., Carvajal, D. y Pérez, J. (2015). Informe de satisfacción estudiantil 2015-1. Santa Marta: Universidad Sergio Arboleda.

Salas, R. (2016). ¿El rediseño curricular sin evaluación curricular es científico? Educación Médica Superior, 30(2).

Saavedra, J. y Medina, C. (2012). Formación para el Trabajo en Colombia. Borradores de Economía, 740.

Stabback, P. (2016). ¿Qué hace a un currículo de calidad? París: UNESCO.

Universidad de Jaén. (2009). Procedimiento, satisfacción, expectativas y necesidades. Sistema de Garantía Interna de Calidad (SGIC). Jaén, España: Universidad de Jaén. 